\title{
Cerebral tuberculosis and secondary epilepsy - Interactions between antiepileptic and antituberculous treatment
}

\author{
Florentina Anca Danciu, Catalina Elena Bistriceanu*
}

Neurology Department, "Prof. Dr. N. Oblu" Neurosurgery Clinical Emergency Hospital, lasi, Romania,

\begin{abstract}
Central nervous system (CNS) involvement represents almost $1 \%$ of all cases of active tuberculosis. It represents a form with a high level of morbidity and mortality in spite of appropriate treatment. There isn't any agreement regarding the duration of the antituberculous treatment. We report a case related to cerebral tuberculomas and another case related to interactions between antiepileptic drugs and antituberculous treatment. The first case involves cerebral tuberculomas discovered on brain imaging (CT scan, cerebral MRI) and secondary epilepsy. The patient did an antituberculous treatment for pulmonary tuberculosis and tuberculous meningitis for one year, consisting of 4 drugs (HRZE). Six months later, he checked into our clinic with a tonic clonic seizure and we discovered tuberculomas on the cerebral MRI. Shortly thereafter we started the treatment with 5 drugs (HRZES) and anticonvulsants. Five months later the MRI showed multiple enhancing lesions described as "a bunch of grapes", potentially matching multiple tuberculomas. The second case shows the interaction between anticonvulsants and antituberculous drugs in concomitant pulmonary tuberculosis and epilepsy with tonic clonic seizures. We measured the concentration of anticonvulsants in the blood during the antituberculous treatment.
\end{abstract}

Keywords: central nervous system, tuberculosis, cerebral tuberculoma, epilepsy, treatment

\section{Introduction}

Tuberculosis is an infectious disease usually caused by Mycobacterium tuberculosis in humans. Although CNS involvement by tuberculosis is seen in all age groups, there is a predilection for younger patients, with 60$70 \%$ of cases occurring in patients younger than 20 years of age [1]. Intracranial tuberculomas are space-occupying masses of granulomatous tissue that result from hematogenous spread from a distant focus of tuberculosis infection.

Received: February 2015; Accepted after review: March 2015; Published: March 2015.

*Corresponding author: Catalina Elena Bistriceanu, MD, Neurology Department, "Prof. Dr. N. Oblu" Neurosurgery Clinical Emergency Hospital, lasi, Romania. Email:catalina nastac@yahoo.com
Histologically, the mature tuberculomas are composed of caseous necrosis center surrounded by a capsule that contains fibroblasts, epithelioid cells, Langhans giant cells, and lymphocytes [2].

The tuberculous granuloma (tuberculoma) is the most common form of parenchymal lesion [3]. Tuberculoma can occur at all age groups. However, its incidence is higher in pediatric population [4]. These are usually located at the cortico-medullary junction and periventricular region, as expected for hematogenous dissemination. They are mostly infratentorial in children, and supratentorial in adults [5, 6]. Parenchymal tuberculomas can be single or multiple, with or without coexisting meningitis [7]. Both the parenchymal and intraventricular tuberculoma may be associated with hydrocephalus [8]. 


\section{Case reports}

Case 1

We report a case of a 25 year-old man who came into our clinic for a generalized tonic clonic seizure. He had no history of convulsions in his childhood and his mother didn't have any problems during pregnancy nor did she have delayed labor. From his personal history we found that 18 months ago he was diagnosed in Denmark with pulmonary tuberculosis (apical right lobe cavern) and tuberculous meningitis.

He presented fever, alteration of general state and weight loss. The cerebrospinal fluid examination revealed 161 leucocytes, increased proteins, a decreased level of glucose and PCR positive for Mycobacterium tuberculosis complex, while the cerebral imaging was normal.
He followed anti-tubercular treatment with 4 drugs: isoniazid, pyrazinamide, ethambutol and rifampin (HRZE) for one year and progressive decreasing doses of corticosteroids and group B vitamin.

Half a year after finishing the treatment, the patient presented a generalized tonic clonic seizure and he came into our clinic for further investigations.

The neurologic examination revealed anisocoria (right>left) with the abolition of pupillary light reflex; without meningeal signs or other pathological findings.

We performed a CT scan with intravenous contrast and it revealed multiple tuberculomas into both sylvian valleys, more obvious on the right one (Figure 1). We confirmed the suspicion with an MRI $(0,4 \mathrm{~T})$ with intravenous contrast.

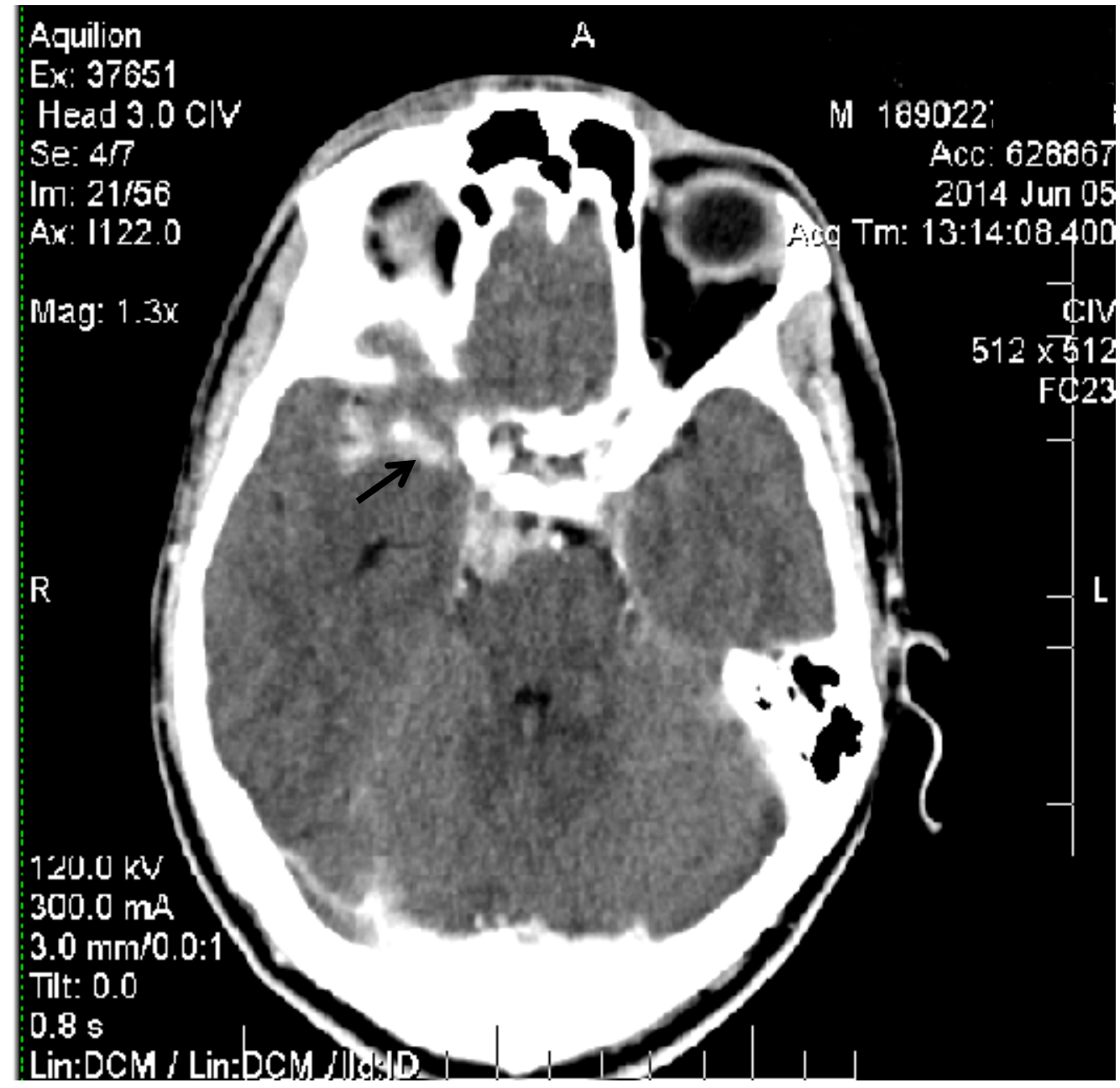

Fig. 1. Axial CT scan with intravenous contrast: multiple enhancing lesions - tuberculomas into both sylvian valleys, more obvious on the right one (arrow). 
The routine laboratory exams and the EKG were normal. The electroencephalogram showed generalized low voltage with polyspikes on left cerebral hemisphere. In hyperventilation, synchronous poly-spikes in both hemispheres could be observed. Then, he went to the Pneumology Department where a lumbar puncture with CSF exam was performed. The tuberculomas diagnosis was confirmed and the antituberculous treatment with 5 drugs (HRZES - isoniazid, pyrazinamide, ethambutol, rifampin and streptomycin) was started. The patient was discharged from the neurology department with recommendations specific for epilepsy together with anticonvulsant drugs during antituberculous treatment. Five months later, the patient was admitted for clinical and preclinical reexaminations.

On a follow-up cerebral MRI $(0,4 \mathrm{~T})$, with intravenous contrast, we found micro-nodular lesions with peripheral enhancement on postcontrast at the circle of Willis, with the aspect of "a bunch of grapes". The formations were more numerous on the right side, being extended not only in the sylvian valley, but also paramedian on the right side of the prepontine cistern (Figure 2).
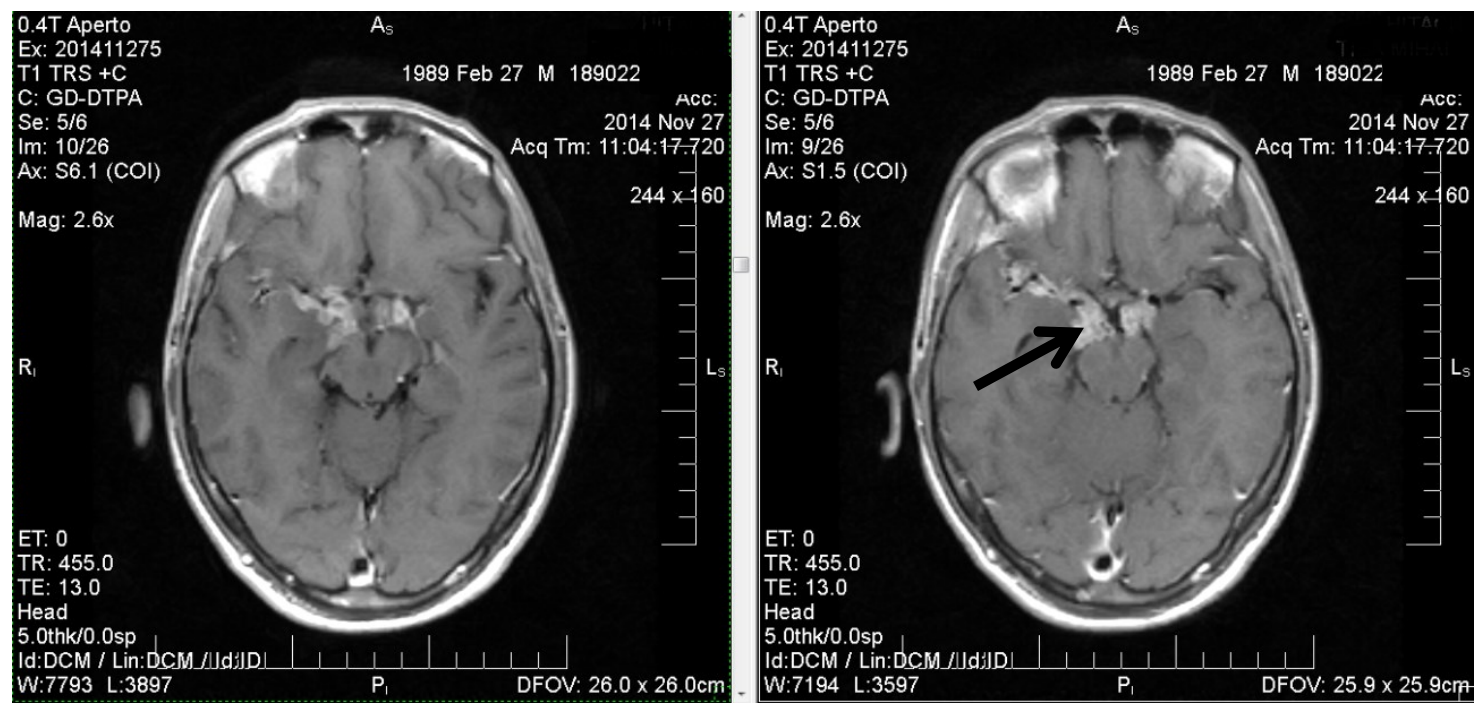

Fig. 2. MRI $(0,4 T)$ with contrast: T1 weighted image of "a bunch of grapes" at the circle of Willis (arrow), more numerous on the right side, being extended not only in the sylvian valley, but also paramedian on the right side of the prepontine cistern.

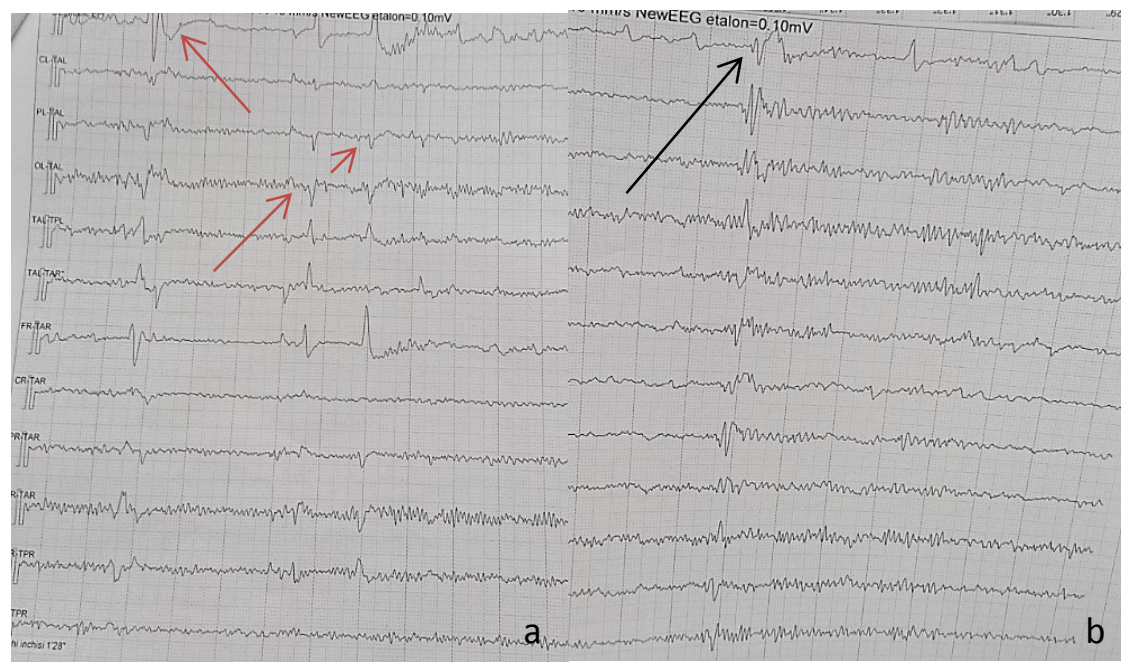

Fig. 3. The electroencephalogram shows: a. isolated spikes in both hemispheres with closed eyes (red arrows); b. in hyperventilation, multiple synchronous sharp waves in both hemispheres (black arrow). 
The electroencephalogram showed multiple polymorphic delta waves and isolated spikes in both hemispheres. In hyperventilation we noticed multiple synchronous sharp waves in both hemispheres (Figure 3). Considering the aspect of the brain imaging, the period of only 5 months of the antituberculous treatment and the EEG findings, it was decided that the patient must continue the recommended treatment. It was necessary to increase the dose of levetiracetam due to the fact that some anti-TB drugs reduce the plasmatic concentration of anticonvulsant drugs.

\section{Case 2}

The second case presents a 43 year-old man that was monitored in our clinic for epilepsy with generalized tonic clonic seizures. The patient was admitted for the evaluation of an anticonvulsant treatment.

From his personal history we found that, one year ago, he was diagnosed with pulmonary tuberculoma (histopathological diagnosis).

At that time, the initiation of the antituberculous treatment raised some problems regarding the contraindications of some anti-TB drugs in epilepsy and the interactions between anticonvulsant drugs and anti-TB treatment. Therefore, the isoniazid was eliminated because its contraindications in epilepsy are well known.

The possibility of the interference of rifampin not only with valproate, but also with lamotrigine was considered. The patient was treated with the maximum doses of both antiepileptic drugs. We decided to determine the blood concentration of valproate and lamotrigine so that the doses could be properly adjusted.

The results showed valproate level 37.5 $\mathrm{mg} / \mathrm{dl}$ (normal value: $50-100 \mathrm{mg} / \mathrm{dl}$ ) and lamotrigine level $4.1 \mathrm{mg} / \mathrm{l}$ (normal value: 3-14 $\mathrm{mg} / \mathrm{l})$. As such, during the antituberculous treatment, the dose of valproate was increased to $3000 \mathrm{mg} / \mathrm{day}$ and the dose of lamotrigine to $250 \mathrm{mg} /$ day.

The patient was monitored after these dosage updates and it was determined that he had the proper blood level of antiepileptic drugs.

Upon finishing the anti-TB treatment, we recommended decreasing the dose of lamotrigine to $200 \mathrm{mg}$ and that of valproate to $2500 \mathrm{mg}$.

\section{Discussions}

Due to its rarity, nonspecific symptoms and radiological findings, intracranial tuberculomas remain a clinical challenge [9].

There is a possibility of future complications with cerebral tuberculomas, even after a correct treatment of the tuberculous meningitis. Our first case revealed that the patient developed cerebral tuberculomas, even after proper antituberculous and corticosteroid treatment for the appropriate time period. One can notice the first case particularity, the tonic clonic seizure complications secondary to cerebral involvement.

The MRI imaging showed "a bunch of grapes" lesions interpreted as multiple tuberculomas. These ring-enhancing lesions represent the conglomeration of multiple small "immature" tubercles into a more identifiable tuberculomatous mass, surrounded by massive edema [10].

Regarding treatment, the Center for Disease Control and Prevention recommends 12 months of treatment for CNS tuberculosis when the Mycobacterium tuberculosis strain is sensitive to all drugs [11]. However, numerous variables can affect the response of the disease to therapy and it has been suggested that treatment duration should be tailored to the radiological response [12].

After 12 months of treatment more than two-thirds of the patients still have contrast enhancing lesions. Although it is not clear if this represents an active lesion or just inflammation, continuing treatment is probably prudent [9]. Total resolution of the tuberculoma is observed when scans demonstrate no enhancing lesions or only an area of calcification [12].

Paradoxically, tuberculomas can develop or enlarge during therapy, leading to new neurological complaints, mainly focal signs or 
symptoms of increased intracranial pressure. A possible explanation could be the massive release of components of the acid-fast bacilli (peptidoglycan and mucopeptide) soon after starting treatment, which leads to inflammatory reaction [13].

The admission of the patient, after finishing the anti-TB therapy one year later, could be explained by immunologic reaction. The complete response after the treatment for tuberculous meningitis excludes the possibility of drug resistance and suggests a reactive phenomenon. T-cell suppression and anergy during active tuberculosis have been well recognized [14]. This phenomenon is related in part to the production of interlukin-10 by peripheral blood T-lymphocytes during active tuberculosis [15]. After completion of tuberculosis therapy, T-lymphocytes no longer produce interlukin-10 [15]. It is known that interlukin-10 down-regulates T-helper cell activity [14]. Therefore, it is not surprising that successful treatment may reinstitute T-cell activity. It means that, over the course of tuberculosis treatment, a heightened immune response may occur despite the vanishing pool of viable mycobacteria. This reversal of

\section{References}

1. Kornienko VN, Pronin IN. Diagnostic Neuroradiology. Berlin Heidelberg Springer Verlag, 2009.

2. Sheller JR, DesPrez RM. CNS tuberculosis. Neurol Clin 1986; 4(1):143-158.

3. Wallace RC, Brutons EM, Beret FF, Leggiadro RJ, Gerald BE, Lasater OE. Intracranial tuberculosis in children. CT appearance and clinical outcome. Pediatric Radiol. 1991; 21(4):241-246.

4. Jamieson $\mathrm{DH}$. Imaging intracranial tuberculosis in childhood. Paediatr Radio 1995; 25(3):165170.

5. Welchman JM. CT of intracranial tuberculoma. Clin Radiol 1979; 30:567-579.

6. Bhargava S, Tandon PN. Intracranial tuberculomas: a CT study. Br J Radiol 1980; 53(634):935- 945.

7. Sze G. Infections and inflammatory diseases. In: Stark DD, Bradley WG Jr. (eds.) Magnetic resonance imaging. St. Louis, MO: CV Mosby, 1988, 316-343. anergy state can explain the paradox of expansion of cerebral tuberculomas despite institution of effective antituberculous drug therapy [16].

In the second case, we illustrated the interactions between antituberculous and anticonvulsant treatments, measuring the blood concentrations of the antiepileptic drugs. We saw the serum fluctuations of lamotrigine and valproate during anti-TB therapy and the necessity of dosage adjustments.

\section{Conclusions}

We conclude that tuberculosis still remains a difficult problem because of its complications and because of the antituberculous treatment characteristics. CNS involvement is a specific topic, if we refer to possible complications and to the duration of treatment.

The association of tuberculosis with epilepsy raises many issues and the main concern of clinicians is to find the proper drug doses and the optimum time frame for curing the patient.
8. Desai K, Nadkarni T, Bhatjiwale M, Goel A. Intraventricular tuberculoma. Neurol Med Chir (Tokyo) 2002; 42:501-503.

9. Monteiro R, Carneiro JC, Costa C, Duarte R. Cerebral tuberculomas - A clinical challenge. Respiratory Medicine Case Reports 2013; 9:3437.

10. Gupta RK, Pandey P, Khan EM, Mittal P, Gujral RB, Chhabra DK. Intracranial tuberculomas: MRI signal intensity correlation with histopathology and localized proton spectroscopy. Magn Reson Imaging 1993; 11(3):443-449.

11. Centers for Disease Control and Prevention. Treatment of Tuberculosis, American Thoracic Society, CDC, and Infectious Diseases Society of America. Treatment of tuberculosis. MMWR 2003; 52(RR11):1-77.

12. Poonnoose SJ, Rajshekhar V. Rate of resolution of histologically verified intracranial tuberculomas. Neurosurgery 2003; 53(4):873879. 
13. Lima MA, Maranhão-Filho $P$, Dobbin $J$, et al. Paradoxical worsening of brain tuberculomas during treatment. Arch Neurol 2012; 69(1):138139.

14. Boussiotis VA, Tsai EY, Yunis EJ, et al. IL-10producing T-cells suppress immune responses in anergic tuberculosis patients. J Clin Invest 2000; 105(9):1317-1325.
15. Garcia M, Vargas JA, Castejon R, Navas $\mathrm{E}$, Durantez A. Flow-cytometric assessment of lymphocyte cytokine production in tuberculosis. Tuberculosis (Edinb) 2002; 82(1):37 - 41.

16. Mohamadnejad M, Bidari A, Moghaddasi M, Mirpour SR. Paradoxal expansion of cerebral tuberculomas during treatment of tuberculous meningitis. Arch Iranian Med 2003; 6(3):219221. 